\title{
Molecular detection of Coxiella burnetti infection in aborted samples of livestock in Iran
}

\author{
Ashraf Mohabati Mobarez ${ }^{1}$, Mohammad Khalili² $^{2}$ Ehsan Mostafavi², and Saber Esmaeili ${ }^{3}$ \\ ${ }^{1}$ Tarbiat Modares University Faculty of Medical Sciences \\ ${ }^{2}$ Affiliation not available \\ ${ }^{3}$ Pasteur Institute of Iran
}

August 28, 2020

\begin{abstract}
Coxiella burnetii is a causative agent of $\mathrm{Q}$ fever which is highly infectious zoonotic disease. C. burnetii has become one of the most important causes of abortion in livestock, which can lead to widespread abortions in these animals. There are very limited studies on the prevalence of C. burnetii infection in cases of animal abortion in Iran. The aim of this study was to investigate C. burnetii in livestock abortion samples in Iran. Abortion samples from cattle, sheep and goat were collected from different parts of Iran and were tested using Real-time PCR targeting IS1111 gene of C. burnetii. In this study, 36 samples (24.66\%) of the 146 collected samples were positive for C. burnetii. The prevalence of C. burnetii was $21.78 \%$ (20 of 94 samples) in sheep samples. Also, 10 of 46 cattle samples (21.74\%) were positive. All six goat abortion samples were positive for C. burnetii. The findings of the study demonstrate that C. burnetii have important role in livestock abortions in Iran and more attention should be paid to $\mathrm{Q}$ fever by the health care system and veterinary organization.
\end{abstract}

\begin{abstract}
Coxiella burnetii is a causative agent of $\mathrm{Q}$ fever which is highly infectious zoonotic disease. C. burnetii has become one of the most important causes of abortion in livestock, which can lead to widespread abortions in these animals. There are very limited studies on the prevalence of $C$. burnetii infection in cases of animal abortion in Iran. The aim of this study was to investigate $C$. burnetii in livestock abortion samples in Iran. Abortion samples from cattle, sheep and goat were collected from different parts of Iran and were tested using Real-time PCR targeting IS1111 gene of C. burnetii. In this study, 36 samples (24.66\%) of the 146 collected samples were positive for C. burnetii. The prevalence of $C$. burnetii was $21.78 \%$ (20 of 94 samples) in sheep samples. Also, 10 of 46 cattle samples $(21.74 \%)$ were positive. All six goat abortion samples were positive for $C$. burnetii. The findings of the study demonstrate that $C$. burnetiihave important role in livestock abortions in Iran and more attention should be paid to Q fever by the health care system and veterinary organization.
\end{abstract}

Keywords : Coxiella burnetii, Q Fever, Abortion, Iran, Livestock.

\section{Introduction}

Coxiella burnetii is a small coccobacillus Gram-negative and obligate intracellular bacterium. C. burnetii is causative agent of $\mathrm{Q}$ fever which is highly infectious zoonotic disease. Based on 16SrRNA sequence analysis, C. burnetii belonged to the Gama-subdivision of Proteobacteria within the Legionellales order and family Coxiellaceae(Eldin et al., 2017). Q fever occurs mainly in two forms of acute and chronic in human. Acute $\mathrm{Q}$ fever is mainly a self-limiting, febrile illness and flu-like disease that is asymptomatic in $60 \%$ of cases (Million and Raoult, 2015). Persistence of C. burnetii infection in humans (in acute and asymptomatic cases) 
can lead to the chronic form of Q fever. Chronic Q fever is extremely serious and dangerous. Endocarditis is the main clinical manifestation of the chronic Q fever that leads to death in over $65 \%$ of the patients, if untreated(Angelakis and Raoult, 2010; Eldin et al., 2017). Livestock are the main reservoirs of C. burnetii. $\mathrm{Q}$ fever in cattle, sheep, and goats is usually asymptomatic but in some cases is associated with pneumonia and reproductive disorders, including abortion, stillbirth, endometritis, and infertility(Guatteo et al., 2011). The main route of transmission to humans is through inhalation of aerosols and dust particles contaminated with $C$. burnetii, which are excreted by infected animals through milk, feces, urine, placenta, fetal fluids, and vaginal discharge(Eldin et al., 2017; Guatteo et al., 2011). People at risk of the disease include farmers, veterinarians, butchers, slaughterhouse workers, farmers, dairy factory workers, laboratory staff, and people in contact with domestic animals, especially during livestock calving(Million and Raoult, 2015). Very easy and fast release capability by aerosol, survival in harsh environmental conditions, very low infectious doses, as well as the high infectivity of the bacterium have made C. burnetii a very serious biological threat to military personnel and civilians(Roest et al., 2013; Van Schaik et al., 2013). In recent years, C. burnetii has become one of the most important causes of abortion in livestock's, which can lead to widespread abortions in animals(Rodolakis, 2009). Also, abortion and the accompanying excretory fluids are one of the main causes of the spread of infection in the environment, which can lead to widespread infection among animals and human populations(Angelakis and Raoult, 2010). There is a very high dose of C. burnetii in amniotic fluid and the placenta during childbirth infected animals. Also, infected aerosols from animal abortions can be transmitted up to several kilometers from the site of the abortion by wind(Tissot-Dupont et al., 2004). Therefore, the role of $C$. burnetii in cases of animal abortion is of great importance both in terms of epidemiology and in the transmission of the disease to humans. In recent years, seroepidemiological studies in animal and human populations in Iran have shown that Q fever is an endemic disease in Iran(Mobarez et al., 2017). However, clinical cases of $\mathrm{Q}$ fever are rarely diagnosed and reported by the health system in Iran(Mobarez et al., 2017). On the other hand, there are very limited studies on the prevalence of $C$. burnetii in cases of animal abortion in Iran. Also, conducting various studies and showing the importance of the disease can cause more sensitivity of the health system and clinical physicians to Q fever in Iran. The aim of this study was to molecular investigation of $C$. burnetii in livestock abortion samples in Iran.

\section{Methods}

2.1. Sample collection: This study was conducted during 2017-2018 with the cooperation of clinical veterinarians as well as the Iranian Veterinary Organization. Livestock aborted samples were collected from different province of Iran and samples included spleen and liver of aborted fetuses, and abortion fluids and cotyledons of aborted animals (cattle, sheep and goat). Sampling was performed in accordance with biological safety conditions. Samples were frozen immediately after obtaining and were transported to the laboratory by maintaining the cold chain. All laboratory works were done under B2 Biological Safety Cabinet.

2.2. Ethical Considerations: This study was approved by the Ethics Committee for Biomedical Research of Tarbiat Modares University (Ethic Code: IR.TMU.REC.1395.510). The Ethics Committee for Biomedical Research of Tarbiat Modares University approved the consent procedure, the proposal and protocol of this study.

2.3. DNA extraction: Genomic DNA was isolated using the Roche High Pure PCR Template Preparation Kit (Roche, Germany), according to the manufacturer's instruction. For tissues samples, $50 \mathrm{mg}$ from each sample used for DNA extraction. Also, $200 \mu \mathrm{L}$ of abortion fluids was used for DNA extraction. All extracted DNA were stored at $-20^{\circ} \mathrm{C}$ until molecular test.

2.4. Real-time Polymerase Chain Reaction (qPCR): Real-time PCR was performed using specific primers and probe sequences targeting IS1111 gene of $C$. burnetii . Real-time PCR reactions were performed using the following reaction mixture: $10 \mu \mathrm{L}$ of 2x RealQ Plus Master Mix for Probe (Ampliqon, Denmark), $900 \mathrm{nM}$ forward primer (AAAACGGATAAAAAGAGTCTGTGGTT), $900 \mathrm{nM}$ reverse primer (CCACACAAGCGCGATTCAT), $200 \mathrm{nM}$ probe (6-FAM-AAAGCACTCATTGAGCGCCGCG-TAMRA) and $4 \mu \mathrm{L}$ of DNA template(Schneeberger et al., 2010). Real-time performed on the Corbett 6000 Rotor-Gene system (Corbett, Victoria, Australia), with a final volume of $20 \mu \mathrm{L}$ for each reaction. The PCR amplification pro- 
gram were 10 minutes at $95^{\circ} \mathrm{C}$, followed by 45 cycles of $15 \mathrm{~s}$ at $94^{\circ} \mathrm{C}$ and $60 \mathrm{~s}$ at $60^{\circ} \mathrm{C}$. DNA of Nine Mile strain (RSA 493), was used as a positive control and double distilled water was used as a negative control. Results were generated with performed using Rotor-Gene@ Q 2.3.5 software (QIAGEN). Samples showing cycle threshold $(\mathrm{Ct})$ values of 36 or lower for C. burnetii IS1111 qPCR assays were considered positive.

\section{Results}

A total of 146 samples of abortions were collected from different parts of Iran, of which 94 samples were from sheep's abortions, 46 samples were from cattle abortions, and 6 samples were from goats' abortions.

In total, 36 samples $(24.66 \%)$ of the 146 collected samples were positive for $C$. burnetii using Real-time PCR. The prevalence of C. burnetii was $21.78 \%$ (20 of 94 samples) in aborted sheep samples. Highest prevalence of C. burnetii was detected in Tehran (54.54\%), Mazandaran (50\%), West-Azarbaijan (40\%) and Hamadan $(38.46 \%)$ provinces, respectively (Table 1$)$.

10 of 46 cattle aborted samples $(21.74 \%)$ were positive for C. burnetii. Highest prevalence of C. burnetii was detected in Tehran (24.32\%), and Alborz (16.67\%) provinces, respectively (Table 2).

All six goats' aborted samples were positive for C. burnetii . Two and four of samples were collected from West-Azerbaijan and Tehran provinces, respectively.

Table 1. The prevalence of $C$. burnetii in sheep abortion samples by using Real-time PCR.

Table 2. The prevalence of C. burnetii in abortion samples of cattle using Real-time PCR.

\section{Discussion}

The present study was conducted to investigate the prevalence of $C$. burnetii in domestic animal abortions in Iran and showed that $24.7 \%$ of the collected samples were positive for C. burnetii using Real-time PCR. According to the results of this study, it is recommended that the effect of $C$. burnetii be considered serious in cases of animal abortion and the possibility of transmission to humans. Despite more than 50 years have passed since the first report of Q fever, but the disease is neglected diseases in Iran. On the other hand, despite the fact that this disease has a very significant seroprevalence among livestock and human populations, but there is no system for registering and reporting and a program for diagnosis and prevention in the human care health system and the veterinary organization in Iran (Mobarez et al., 2017). Therefore, conducting more studies on suspected patients and conducting molecular epidemiological research will shed light on the epidemiological situation of fever in Iran and will encourage more attention to the human and animal health system towards this disease. It was recently reported that about $31 \%$ of culture-negative endocarditis caused by C. burnetii in Iran (Moradnejad et al., 2019).

In current study, $21.8 \%$ sheep's aborted samples were positive for of $C$. burnetii . Highest prevalence diagnosed Tehran (54.5\%), Mazandaran (50\%), West-Azarbaijan (40\%) and Hamadan (38.5\%) provinces, respectively. Prevalence in our study was higher than other similar studies in Iran, so that the molecular prevalence of $C$. burnetii in sheep's abortion in Mashhad county (north-east of Iran), Sistan region (south-eastern of Iran) and Fars province (southern of Iran) were reported 17.3\%, 16.6\% and 2.7\%, respectively (Abiri et al., 2016; Heidari et al., 2018; Mahdavi Roshan et al., 2018). One of the reasons for the high prevalence in our study was the using of a much more sensitive method (Real-time PCR) for detection of $C$. burnetii . Other reasons include different study areas and increased prevalence over time. In other countries, different rates were reported; $33.6 \%$ in Egypt(Selim et al., 2018), 2-11\% in Turkey(Gunaydin et al., 2015; Kılıç et al., 2016), 21.5\% in Italia(Parisi et al., 2006), 47.6\% in Hungary(Kreizinger et al., 2015) and $44.4 \%$ in Switzerland(Magouras et al., 2017). Based on recent study in Iran, shedding of C. burnetii to milk was significantly high (35.7\%) among sheep with abortion history(Esmaeili et al., 2019). Therefore, it seems that abortion in sheep due to $C$. burnetii should be given more attention.

In our study, $21.7 \%$ of cattle aborted samples were positive for $C$. burnetii . Highest prevalence of $C$. burnetiiwas detected in Tehran (24.3\%), and Alborz (16.7\%) provinces, respectively. However, the very small number of sample s from other provinces made it difficult to compare in our study. In a study in Mashhad 
county, $25 \%$ of cattle fetuses were positive for C. burnetii (Abiri et al., 2016). In other countries, prevalence of C. burnetii in cattle abortions were $4 \%$ in Turkey (Gunaydin et al., 2015), 11.6\% in Italy (Parisi et al., 2006), 25.9\% in Hungary (Kreizinger et al., 2015) and 35\% in Cyprus (Cantas et al., 2011). Also, C. burnetii was detected $33.3 \%$ in milk samples of cattle with abortion history in Iran (Esmaeili et al., 2019). According to the findings of this study and other similar studies in Iran and other countries, it is recommended to take control measures against this bacterium in order to prevent of cattle abortions in Iran.

The findings of the study demonstrate that $C$. burnetii have important role in livestock abortions in Iran and more attention should be paid to $\mathrm{Q}$ fever by the health care system and veterinary organization. Also, necessary training in the prevention of this disease should be provided to ranchers and at-risk people.

\section{Acknowledgment}

This work was supported financially by Tarbiat Modares University (Tehran, Iran), Pasteur Institute of Iran and Centre for Communicable Diseases Control in Ministry of Health (grant 810),

and also, Iranian National Scientific Foundation (INSF; Contracted No.91004716). We would like to express our gratitude to the veterinary clinicians of large animal for their helps in sampling.

\section{Declaration of Competing Interest}

The authors declare that no conflict of interest exists.

\section{Data Availability Statement}

The data that support the findings of this study are available from the corresponding author upon reasonable request.

\section{Reference}

Abiri, Z., Khalili, M., Rad, M., Sharifi, H., 2016. Detection of Coxiella burnetii in aborted fetuses of cattle and sheep using polymerase chain reaction assay in Mashhad city, Iran. International Journal of Enteric Pathogens 4, 9-33170.

Angelakis, E., Raoult, D., 2010. Q fever. Veterinary Microbiology 140, 297-309.

Cantas, H., Muwonge, A., Sareyyupoglu, B., Yardimci, H., Skjerve, E., 2011. Q fever abortions in ruminants and associated on-farm risk factors in northern Cyprus. BMC Veterinary Research 7, 1-7.

Eldin, C., Melenotte, C., Mediannikov, O., Ghigo, E., Million, M., Edouard, S., Mege, J.-L., Maurin, M., Raoult, D., 2017. From Q fever to Coxiella burnetii infection: a paradigm change. Clinical Microbiology Reviews 30, 115-190.

Esmaeili, S., Mohabati Mobarez, A., Khalili, M., Mostafavi, E., 2019. High prevalence and risk factors of Coxiella burnetii in milk of dairy animals with a history of abortion in Iran. Comparative Immunology, Microbiology and Infectious Diseases 63, 127-130.

Guatteo, R., Seegers, H., Taurel, A.-F., Joly, A., Beaudeau, F., 2011. Prevalence of Coxiella burnetii infection in domestic ruminants: a critical review. Veterinary Microbiology 149, 1-16.

Gunaydin, E., Mustak, H.K., Sareyyupoglu, B., Ata, Z., 2015. PCR detection of Coxiella burnetii in fetal abomasal contents of ruminants. Kafkas Universitesi Veteriner Fakultesi Dergisi 21, 69-73.

Heidari, S., Derakhshandeh, A., Firouzi, R., Ansari-Lari, M., Masoudian, M., Eraghi, V., 2018. Molecular detection of Chlamydophila abortus, Coxiella burnetii, and Mycoplasma agalactiae in small ruminants' aborted fetuses in southern Iran. Tropical Animal Health and Production 50, 779-785.

Kılıç, A., Kalender, H., Koç, O., Kılınç, Ü., Irehan, B., Berri, M., 2016. Molecular investigation of Coxiella burnetii infections in aborted sheep in eastern Turkey. Iranian Journal of Veterinary Research 17, 41-44. 
Kreizinger, Z., Szeredi, L., Bacsadi, Á., Nemes, C., Sugár, L., Varga, T., Sulyok, K.M., Szigeti, A., Ács, K., Tóbiás, E., 2015. Occurrence of Coxiella burnetii and Chlamydiales species in abortions of domestic ruminants and in wild ruminants in Hungary, Central Europe. Journal of Veterinary Diagnostic Investigation 27, 206-210.

Magouras, I., Hunninghaus, J., Scherrer, S., Wittenbrink, M., Hamburger, A., Stärk, K., Schüpbach-Regula, G., 2017. Coxiella burnetii Infections in Small Ruminants and Humans in S witzerland. Transboundary Emerging Diseases 64, 204-212.

Mahdavi Roshan, H., Saadati, D., Najimi, M., 2018. Molecular detection of Brucella melitensis, Coxiella burnetii and Salmonella abortusovis in aborted fetuses of Baluchi sheep in Sistan region, south-eastern Iran. Iranian Journal of Veterinary Research 19, 128-132.

Million, M., Raoult, D., 2015. Recent advances in the study of Q fever epidemiology, diagnosis and management. Journal of Infection and Public Health 71, S2-S9.

Mobarez, A.M., Amiri, F.B., Esmaeili, S., 2017. Seroprevalence of Q fever among human and animal in Iran; A systematic review and meta-analysis. PLoS Neglected Tropical Diseases 11, e0005521.

Moradnejad, P., Esmaeili, S., Maleki, M., Sadeghpour, A., Kamali, M., Rohani, M., Ghasemi, A., Bagheri Amiri, F., Pasha, H.R., Boudagh, S., Bakhshandeh, H., Naderi, N., Ghadrdoost, B., Lotfian, S., Dehghan Manshadi, S.A., Mostafavi, E., 2019. Q fever endocarditis in iran. Scientific reports 9, 1-7.

Parisi, A., Fraccalvieri, R., Cafiero, M., Miccolupo, A., Padalino, I., Montagna, C., Capuano, F., Sottili, R., 2006. Diagnosis of Coxiella burnetii-related abortion in Italian domestic ruminants using single-tube nested PCR. Veterinary Microbiology 118, 101-106.

Rodolakis, A., 2009. Q Fever in dairy animals. Annals of the New York Academy of Sciences 1166, 90-93.

Roest, H.I., Bossers, A., van Zijderveld, F.G., Rebel, J.M., 2013. Clinical microbiology of Coxiella burnetii and relevant aspects for the diagnosis and control of the zoonotic disease Q fever. Veterinary Quarterly 33, 148-160.

Schneeberger, P.M., Hermans, M.H., van Hannen, E.J., Schellekens, J.J., Leenders, A.C., Wever, P.C., 2010. Real-time PCR with serum samples is indispensable for early diagnosis of acute Q fever. Clinical and Vaccine Immunology 17, 286-290.

Selim, A., Ali, A.-F., Moustafa, S.M., Ramadan, E., 2018. Molecular and serological data supporting the role of Q fever in abortions of sheep and goats in northern Egypt. 125, 272-275.

Tissot-Dupont, H., Amadei, M.-A., Nezri, M., Raoult, D., 2004. Wind in November, Q fever in December. Emerging Infectious Diseases 10, 1264.

Van Schaik, E.J., Chen, C., Mertens, K., Weber, M.M., Samuel, J.E., 2013. Molecular pathogenesis of the obligate intracellular bacterium Coxiella burnetii. Nature Reviews Microbiology 11, 561-573.

\section{Hosted file}

Table 1.docx available at https://authorea.com/users/354570/articles/478097-moleculardetection-of-coxiella-burnetti-infection-in-aborted-samples-of-livestock-in-iran

\section{Hosted file}

Table 2.docx available at https://authorea.com/users/354570/articles/478097-moleculardetection-of-coxiella-burnetti-infection-in-aborted-samples-of-livestock-in-iran 